\title{
METHODOLOGICAL APPROACH FOR DEVELOPING A KMS: A CASE STUDY
}

\author{
Anna Grimán, Teresita Rojas, María Pérez \\ Departamento de Procesos y Sistemas, Universidad Simón Bolívar, Venezuela - \\ \{agriman, trojas, movalles\}@usb.ve
}

\begin{abstract}
This article describes the construction of a Knowledge Management System (KMS) by applying fundamental concepts and principles of Software Engineering, such as process, models, methodology, technology and architectures, among others. They are applied to the field of Knowledge Management as a means of improving the process of developing applications in this domain, in an endeavor to increase its effectiveness.
\end{abstract}

This paper's main contribution is a methodological approach progressing from knowledge processes to a set of appropriate systems. The use of modeling techniques and technologies that may prove helpful to the Software Engineer are suggested as part of this approach, while the technological, social and organizational aspects to be considered are highlighted. This proposal was evaluated through the development of a Knowledge Management System for a Venezuelan organization - a research center.

The evaluation proved the effectiveness of the methodological aspects proposed. These may be of use as inputs for some Learning Software Organizations dedicated to the development of this type of system.

Keywords: Knowledge Management, Knowledge Management System, Methodological Approach, Methodology.

\section{INTRODUCTION}

Knowledge Management (KM) deals with the critical problems of organizational adaptation, survival and competence in the face of increasingly frequent changes in the environment. It includes organizational processes aimed at achieving a synergic combination between the capacity of information technologies (IT) to process data and information, and the creative and innovative capacity of human beings [19]. KM enables an organization to increase its ability to create and exchange knowledge both within itself (internal learning) and with other organizations (external learning) by using information and communication technologies (Internet, intranets, extranets, among others) [4].

This is an effective way of consciously designing and developing a Knowledge Management System (KMS) that supports the integration of all 
knowledge-related aspects.

Developing this type of system is not a simple task, it cannot be carried out in the traditional manner. It warrants the involvement of some systemically related variables: people, technology and the organizational structure. Gold et al. also call them key infrastructures for maximizing social capital [8]. Grover and Davenport [11] identify them as key domains for pragmatic research into $\mathrm{KM}$ processes. These variables have a special implication when it comes to managing knowledge (for instance, creation of a new organizational culture, designation of roles and responsibilities, creation of an appropriate, flexible technical platform, high degree of Management commitment), which is why they must not be lost sight of when developing a system that supports such management. Gold et al. say that emphasis in vision statement and value systems should be placed on the components of the organization that encourage effective $\mathrm{KM}$ process to occur [8].

These considerations mean that development methodologies for KMS should not focus on product alone but must also take into account process variables as critical management factors for ensuring success. One such aspect that stands out in particular is the need to represent knowledge processes so they can be traced to adequate information systems (IS) without losing their semantic richness.

Becerra et al. [2] say the effectiveness of $\mathrm{KM}$ process is influenced by the particular context in which the knowledge is being used.

These reasons prompted this research, the objective of which is to test a set of methodological issues that includes the main socio-technical aspects inherent to $\mathrm{KM}$, and sees it as a subsystem within a larger system -the organization. This proposal pinpoints the necessary activities, deliveries, methods, techniques, tools and roles that are suitable. It begins by looking at and exploring existing $\mathrm{KM}$ processes and proposes a way to restructure them with support from IT. This triggers a series of information systems (IS) that together make up the platform on which the processes for generating, coding and transferring knowledge within the organization are managed.

This article presents a brief definition and characterization of KMS, underlines the main aspects of the methodological proposal for $\mathrm{KM}$, presents the application of this proposal to a case study, as well as the analysis of the results obtained from that application, and ends with the conclusions and recommendations derived from this research.

\section{KNOWLEDGE MANAGEMENT SYSTEMS}

For O'Brien [22] KMS are systems that support the creation, organization and dissemination of knowledge about the business within a company, KMS support includes processes, procedures, 
patents, reference papers, formulas, best practices, forecasts, etc. These systems use a wide variety of IT to gather and edit information, assess its value, disseminate it within the organization and apply it to the business processes. Some technologies like Internet and intranets websites, groupware, data mining, knowledge bases, discussion forums and videoconferencing enable the knowledge to be stored and distributed [22]. These technologies facilitate inputting and feeding back Organizational Memory that contains the interpretations through which past knowledge is taken to the present to support activities by re-using knowledge; through purchase, maintenance, retention and retrieval [25][1][3][6][16][23] [29].

This is why these systems must not be confused with Expert Systems or Artificial Intelligence. According to Laudon and Laudon [18], this technology is only a set within the IS designed to support KM. They are particularly useful for capturing and coding knowledge. Therefore, this technology may or may not be part of a KMS.

According to Mc Lure [20] a KMS can be used to code, store and distribute the company's knowledge base. $\mathrm{He}$ believes that KMS can be used as a knowledge repository provided that the knowledge can be coded. KMS also support social capital by establishing structural links between people, regardless of time and geographical barriers, thereby improving the capacity to combine and exchange intellectual capital.

These definitions establish the role of KMS in knowledge generation, coding and transfer processes [5]. According to O'Brien [22], many organizations are developing KMS to manage Organizational Learning and knowhow.

KMS create organizational learning cycles (learning loops) where the creation, dissemination and application of knowledge produce an adaptive learning process within a company [22].

O'Brien [22] says KMS is able to give knowledge workers rapid feedback, foster changes in employees' behavior and significantly improve business performance. As the organizational learning process progresses and the knowledge base expands, Learning Organization integrates its knowledge into its business processes, products and services.

Turban et al. [28] also believe that the new challenge of $\mathrm{KM}$ requires IS departments to begin providing systems that support it. They feel that the activities that should be largely supported by these systems are Knowledge identification (Determination of critical knowledge in decisionmaking); Discovery and analysis of knowledge (Use of search instruments, databases and data mining); Establishment of organizational knowledge bases (Organizational Memory and best practices must be stored in a properly indexed and maintained knowledge 
base) and; Use and distribution of knowledge (Definition of a suitable audience and placement of technologies to expedite delivery of knowledge as and when it is needed).

There are actually many cases, initiatives and projects where KM has been applied, for instance by Benetton (Italia), General Electric (USA), Netscape (USA), Frito-Lay (USA), Dow Chemical (USA), Scandia (Suzie), 3M (USA), Hewlett-Packard, Arthur Andersen, Ernst \& Young, Microsoft (USA), Xerox (USA), IBM (USA), British Petroleum, to name but a few [5][27][26].

The majority of these experiences reflect the application and usefulness of IT at the service of KM. In this research they were analyzed in an endeavor to find methodological aspects that might be reused in similar development efforts [9].

Given the foregoing, it is convenient to establish a process that involves the socio-technical aspects underlying KM, using a systemic approach, process modeling, and without losing sight of its place in the organizations. This approach involves managing technology, organizational culture and change. This research arose in response to this need.

\section{METHODOLOGICAL APPROACH FOR KNOWLEDGE MANAGEMENT IN ORGANIZATIONS}

In this research a set of methodological issues including the different aspects involved and promoting appropriate IT support was proposed [10].

This approach proposes a development process (including phases and workflows), combined with a series of workers, models and check-list to generate important deliveries or products: a KMS for the organization and the analysis and design specifications required for its documentation and maintenance.

This methodological proposal arose from the combination and adaptation of models and methodologies that are widely used in software development and Business Process Reengineering, such as: Socio-Technical Model for Knowledge Management (STMKM) [12], Rational Unified Process (RUP) [17], Business Process Engineering with Object Technology (BPEOT) [15], Microsoft Solution Framework (MSF) [21], and Knowledge Management Framework Methodology (KMFM) [7].

RUP and MSF were chosen for their iterative/incremental development process and because they handle software product quality criteria. Additionally, both methodologies are widely used in industry. This criterion is upheld by a study conducted by Firestone [7] in which the author presents evidence of the advantages of an iterative/incremental development process for the KM domain. STMKM was considered in this study as it covers different aspects of $\mathrm{KM}$ that are compatible with the proposals made in 
this approach. Lastly, BPEOT was selected for being a business-centric methodology that has already proven the effectiveness of a set of UML models applied to the understanding of business processes [15].

Lastly, KMFM was analyzed because it is a specific methodology for $\mathrm{KM}$, inspired by Unified Process [8].

Although for the purpose of this study, only those methodologies and models were considered, this does not mean one must disregard, in subsequent refinements, the possibility and need to consider other methodologies issues that might enrich the base of practices applicable to KM.

The features of each of them were analyzed in accordance with needs to monitor the problem at hand (see Table 1).

\begin{tabular}{|c|}
\hline FEATURES \\
\hline 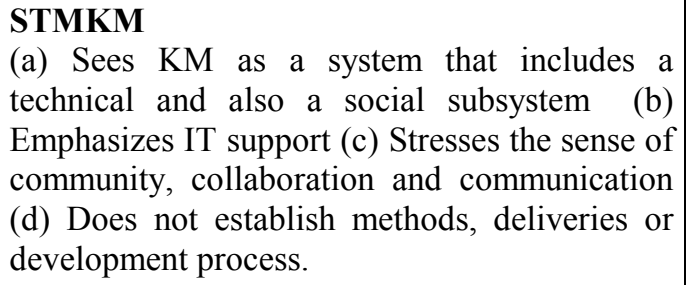 \\
\hline $\begin{array}{l}\text { KMFM } \\
\text { (a) This was specifically proposed for the KM } \\
\text { domain (b) Sets patterns for tasks and } \\
\text { deliverables (c) Aimed at development of } \\
\text { simulations of KM solutions that will be } \\
\text { implemented (d) Does not consider critical } \\
\text { success factors to be managed during the } \\
\text { process. }\end{array}$ \\
\hline
\end{tabular}

\section{MSF}

(a) Includes milestones (b) Is systemic (c) Emphasizes vision and scope rather than requirements (d) Milestones are user-oriented and not development-oriented (e) Includes Version Management (f) Proposes the use of roles (g) Iterative (h) Proposes Business Modeling (i) Does not include Change Management (j) Does not include Project Management (k) Does not specify modeling techniques or tools (l) Has a software-oriented application level.

\section{BPEOT}

(a) Proposes commonly use cases as a way of identifying business processes (b) Proposes the use of models to describe the company (c) Uses an object-oriented approach to describe the company's internal functions (d) Proposes IS support (e) Includes Continuous Improvement (f) Has a business-oriented application level (g) Does not include Change Management or Project Control (h) Created for a general domain (it was not designed specifically for KM).

RUP

(a) Proposes iterative development (b) Promotes requirements management (c) Uses components-based architectures (d) Facilitates visual modeling of software (e) Checks software quality (f) Includes Change and Risk Management (g) Proposes use case-driven development (h) Includes process configuration (i) Proposes a high level of reuse (j) Facilitates team learning $(\mathrm{k})$ Allows the product to have a global quality (l) Manages the support of tools (m) Its architecture is based on $4+1$ views: logic, implementation, process, deployment and use case (n) Has a software-oriented application level.

Table 1. Analysis of features of the Methodologies

The methodological base applied here for the development of KM was obtained from the analysis of the strengths of each of these models and 
methodologies.

Three-way systemic thinking was applied in this methodological proposal: (1) the premise is that a methodology for KM cannot be a straight-jacket for the organization, but should adapt to the elements and characteristics that differentiate it; (2) $\mathrm{KM}$ is considered a complex system immersed in a larger one - the organization - where the former integrates the technical and social subsystems and must maintain a systemic relationship with them; and (3) the idea of Learning Organizations, which are based on the organization's focus as an open system, was taken as a reference framework.

These three assumptions influenced the fact that the methodological aspects to be proposed might include a set of critical success factors that reflect this systemic thinking and boost the success of the development.

\subsection{Critical success factors}

The methodological proposal put forward includes a series of factors that are decisive for successful management. These factors are decisive for the success of the KM initiative and are proposed under a flexible perspective. In other words, the organization must determine how they are to be implemented. These factors (see Table 2) are broken down into five types: human (implying changes in people), organizational (involving changes in the organization's structure), humanorganizational (involving changes in the organizational culture), technological (changes in techniques and tools) and management (implying actions by senior and middle managers in the organization).

\section{CRITICAL SUCCESS FACTORS}

Human: Trust, Relationships, Collaboration, Cooperation and Communication, Sense of Community, Learning Efficacy, Creativity, Imagination and Human Creativity

Organizational: Well-defined Roles and Responsibilities, Management of the Knowledge Market

Management: Risk Management,
Commitment towards the Community, Strategic Alignment.

Human-Organizational: Motivation, Leadership, Shared Vision, Systemic Thinking, Collective and Generative Learning, New Patterns of Thinking, Recognition, Promotion and Reward for Sharing Knowledge.

Technological: Tangible Products, Technology Support, Organizational Memory, Qualitative and Quantitative Measurements, Accessibility, A New Technical Architecture.

Table 2. Critical success factors for KM

The idea behind this classification is not to establish a hierarchy but to emphasize that they are aimed at the three broad vertices mentioned above: people, structure and technology. It was proposed that the three aspects be managed holistically in order to guarantee the success of KM; however, a multi-layer approach can be used, which starts with managing the humanorganizational-management layers in order to foster a sufficiently favorable climate for it to be able to be supported by technology later on. 


\subsection{Workers: the roles needed}

The main roles proposed for the $\mathrm{KM}$ project are: line workers or knowledge workers, KM workers, knowledge project workers, senior knowledge executives. Among these senior executives, the Chief Knowledge Officer $(\mathrm{CKO})$ warrants special mention and that person's responsibilities will be: to obtain knowledge and to learn; to design, implement and supervise a knowledge infrastructure in the company; to handle relationships with external information and knowledge suppliers and to negotiate contracts with them.

Furthermore, that person must provide critical inputs to the process of creating knowledge and its use within the company and endeavor to improve such processes if necessary; design and implement the company's knowledge coding approaches; measure and manage the value of knowledge; manage the organization's professional knowledge managers, give them a sense of community, set professional standards and manage their careers; drive the development of a knowledge strategy, focusing the company's resources on the type of knowledge that most needs to be managed and the knowledge processes that have the most marked differences between their needs and their current capacity; build a knowledge culture; create a KM infrastructure; make all the financial arrangements; understand how people learn and share their knowledge and experience with colleagues; provide processes from which new knowledge can be learned and shared; provide rewards and incentives to contribute to the organizational knowledge base; transform individual learning into organizational learning.

Once these factors have been established for consideration by the organization, a series of activities that enable the project to be developed and monitored has to be defined.

\subsection{Process for KM}

The process proposed is an iterative one organized in 4 phases, as can be seen from Figure 1.

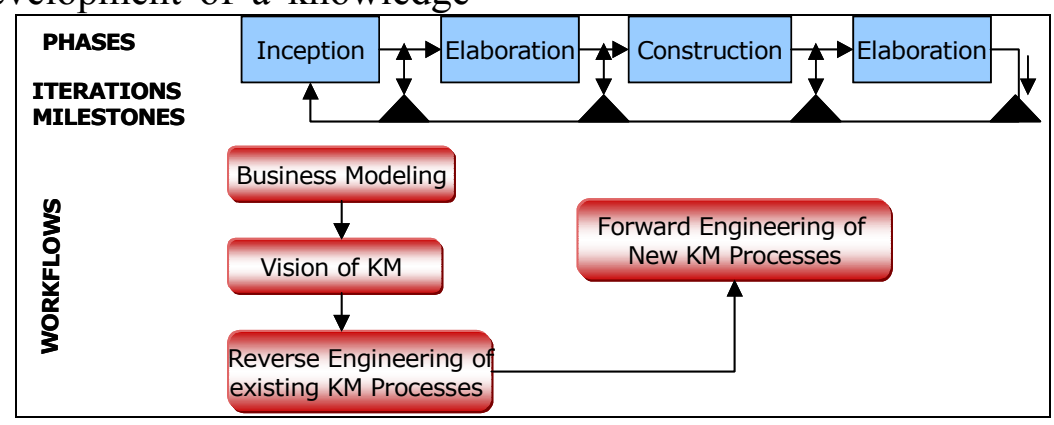

Figure 1. Process proposed for KM 


\subsection{Development cycle for $\mathrm{KM}$}

This process provides a way of evaluating the progress of the $\mathrm{KM}$ project, since a project must converge in each iteration. The milestones provide points in time in which a decision must be taken to proceed, abort or change the course. The sequence of iterations must be partitioned and organized in accordance with the specific short term objectives. Progress is measured by the number of use cases completed, characteristics completed, risks eliminated, etc. The 4 phases of the KM development cycle are [9]:

\subsubsection{Inception}

This phase consists of: defining the project's scope, the vision of $\mathrm{KM}$ development and the context of the business. The Inception phase concludes with the milestone Specification of KM Objective.

\subsubsection{Elaboration}

This phase consists of planning the activities necessary and the resources required. This is when the existing processes are specified and the architecture of the $\mathrm{KM}$ processes is designed. The Elaboration phase concludes with the milestone $K M$ Architecture.

\subsubsection{Construction}

During this phase the KM processes are implemented according to the Vision and Architecture. The development products are ready to be introduced in the organization. The Construction phase ends with milestone Initial Operating Capacity.

\subsubsection{Transition}

This is the transition of the processes and KMS to the organization and includes implementation, training, support and maintenance until the direct users are satisfied. The Transition phase culminates with the milestone Version of the Product and Processes.

Each of these phases involves executing one or more workflows to achieve the goals and attain the milestones that enable the iterations to be monitored and their culmination determined.

\subsection{Workflows}

A workflow is a sequence of activities that produces a result with an observable value [17]. The methodological proposal consists of 4 core workflows and 3 support workflows, as can be seen in Figure 1.

The core workflows are the core of KM development and are specific to it. They are:

\subsubsection{Business Modeling}

Its purpose is to understand the structure and dynamic of the organization, ensure a common understanding of the organization and describe the business processes that will be supported by the KM initiative. This modeling calls for a use case and object-oriented approach. Some of the related activities are: capturing a common vocabulary; pinpointing the actors and use cases in the business; 
describing the use cases; identifying and describing business workers and entities; structuring the Business use case model; structuring the Business object model.

Its deliveries are: the Business use case model, the Business object model.

\subsubsection{Vision of $\mathrm{KM}$}

Its objective is to develop the vision for the KM initiative in terms of specifying the objective. This vision covers several steps: aligning the organization's strategic goals with the goals set by $\mathrm{KM}$; seeking an understanding of the existing business functions, in order to pinpoint those needing support from $\mathrm{KM}$; study the demands of knowledge of the workers in the organization; compare the efforts of $\mathrm{KM}$ in other organizations through a benchmarking activity.

The delivery of this flow is: Specification of the Objective of KM.

\subsubsection{Reverse Engineering of existing KM processes}

Its purpose is to provide an understanding of the current situation of $\mathrm{KM}$, determine what should be done to change these processes, train management for change and measure the use cases to be changed. A use case and object-oriented approach is suggested for executing this workflow. The activities involved are: pinpointing actors and use cases for KM processes; describing the use cases; building the use case model for KM processes; prioritizing use cases; selecting metrics.
The flow's deliveries include: use case model for the existing process, list of critical use cases, vision of the new processes, metrics.

\subsubsection{Forward Engineering of new KM processes}

Its objective is to implement the new processes and the improved processes based on the Specification of the KM Objective and the metrics evaluated. In order to execute this workflow, a use case and object-oriented approach is suggested (e.g. RUP). The activities involved are: building a real object model; developing the IS (KMS); installing the new process and IS; checking new processes; building the team model.

The related deliveries are: use case model, ideal object model, portfolio of IS projects, KMS Prototype and its architecture.

Even though the support workflows for KM development are not the essence of this development, they are highly relevant for its monitoring and success. They are:

\subsubsection{Project Management}

Their purpose is to provide a reference framework to manage the projects, provide practical standards for planning, executing and monitoring projects and providing a reference framework for Risk Management. In order to enable the progress of the development to be monitored, each phase of the process covers a specific milestone to be attained if this is not achieved and the 
following must be considered: 1) make a new iteration, 2) re-plan the project, 3) cancel it.

\subsubsection{Configuration and Change Management}

Its purpose is to follow up and maintain the integrity of the project's assets and how they evolve in the face of change.

As far as organizational change is concerned, this workflow takes into account a set of techniques and dynamics that enable a knowledgesharing culture to be fostered and rewarded.

The effectiveness of the knowledge being incorporated in the KMS can be measured qualitatively (precision, accuracy, etc) and quantitatively (direct commercial value of the processes, staff costs, support costs, time-to-market, number of visits to knowledge sites on the intranet, number of recurring visits to the site, lessons learned documented, etc.), depending on the organization's current phase of KM application [13]. One of the measurement/valuation methodologies discussed by Housell and Bell [14] should also be followed as well: cost, income or market approach, real options and knowledge value added.

\subsubsection{Environment}

Its purpose is to support the $\mathrm{KM}$ initiative, from the point of view of processes and tools. This support includes: selection and acquisition of tools, adaptation of tools, process configuration, process improvement, training, technical service to improve the process.

As mentioned above, the phases of the iterative development cycle evolve towards the desired product, while the workflows provide activities, players or roles and deliverables that guarantee fulfillment of the plans proposed for each phase. One of the workflows of particular interest presented in this section is Forward Engineering of new processes since there are techniques and other related aspects (tools, team model, use cases for KMS and metrics) that can be suggested [9].

\subsubsection{IT supporting KM strategies}

In the Forward Engineering phase it is particularly important to complement the KM strategies to be implemented with a set of ITs that support their effectiveness (See Table 3).

\begin{tabular}{l|l}
\hline \multicolumn{1}{c|}{ Strategy supporting KM } & \multicolumn{1}{c}{ IT } \\
\hline - Record data on experiences. & $\begin{array}{l}\text { Data Base Management Systems, Data Mining, } \\
\text { Data Warehousing, Knowledge Bases. }\end{array}$ \\
\hline - Establish structured and unstructured, formal and informal, & $\begin{array}{l}\text { Virtual collaborative environments, groupware, } \\
\text { intranets, extranets, practice communities, } \\
\text { shareware groups. }\end{array}$ \\
mechanisms to generate or produce knowledge. & \\
- Implement self-managed work groups to of re-dimension the \\
role of management.
\end{tabular}




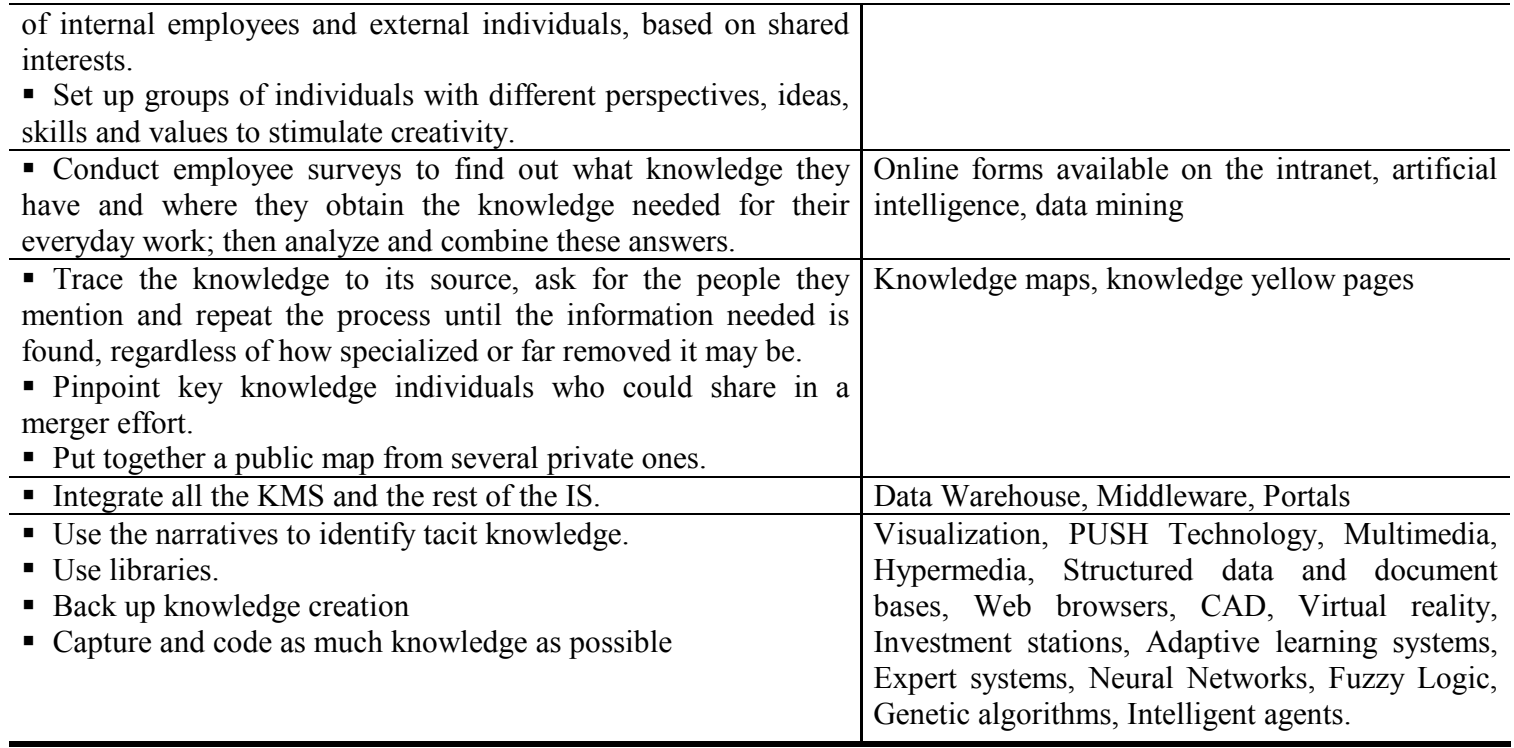

Table 3. IT supporting KM strategies

\subsubsection{Use case check-list for a KMS}

In this phase too we recommend a set of Use Cases that represent common tasks for the KMS aimed at supporting both knowledge processes (See Table 4) and knowledge managers' and senior executives' (e.g. Chief Knowledge Officer) own processes (See Table 5).

\begin{tabular}{l|l}
\hline $\begin{array}{l}\text { KNOWLEDGE } \\
\text { PROCESS }\end{array}$ & KMS USE CASES \\
\hline Generation & $\begin{array}{l}\text { Cataloguing and following up previously acquired and business process-related data } \\
\text { business knowledge, information and data bases. } \\
\text { Receipt of data, information and knowledge sent by e-mail and automatic alert and } \\
\text { mechanisms and updates. } \\
\text { Retrieval through computer based queries, data, information and knowledge related } \\
\text { to: planning, descriptions, cause-effect, time series predictions and forecasts, assets. } \\
\text { Preparation of data, information and knowledge for analytical modeling. } \\
\text { Modeling, including revision, formulation and reformulation of models. } \\
\text { Evaluation of models and demand for knowledge in competition using appropriate } \\
\text { analytical techniques, data and validation criteria. } \\
\text { Evaluation of test results and comparison of models and requests for knowledge in } \\
\text { competition. }\end{array}$ \\
\hline Acquisition & $\begin{array}{l}\text { Cataloguing and following up external previously acquired and business process- } \\
\text { related business knowledge, information and external data bases } \\
\text { Organization of data, information and external knowledge requested and dispatched }\end{array}$ \\
\hline
\end{tabular}




\begin{tabular}{l|l}
\hline & $\begin{array}{l}\text { from outside sources. } \\
\text { Purchase of data, information and external knowledge requested. } \\
\text { Extracting, re-formatting, cleaning up, changing, organizing and loading data, } \\
\text { information and requests for knowledge acquired from outside sources. } \\
\text { Assessment of test results and comparison of models and requests for knowledge in } \\
\text { competition. }\end{array}$ \\
\hline coordination & $\begin{array}{l}\text { Storage of outputs from searches, filters, receipts, conclusions and other generation } \\
\text { activities in the data, information and knowledge stores accessible through } \\
\text { electronic query systems. } \\
\text { Evaluation of models and requests for knowledge in competition using the } \\
\text { appropriate analytical techniques, data and validation criteria. } \\
\text { Updating all data, information and knowledge repositories to maintain consistency } \\
\text { with the changes made. }\end{array}$ \\
\hline Transfer & $\begin{array}{l}\text { Publish and disseminate data, information and knowledge through the company's } \\
\text { intranet. } \\
\text { Load data, information, knowledge and updates within the company's repository } \\
\text { and provide access to the company’s groupware tools. } \\
\text { Query-based search/retrieval. } \\
\text { Use of e-mail to request assistance from personal networks. }\end{array}$ \\
\hline
\end{tabular}

Table 4 . KMS use cases for knowledge processes

Source: Adapted from [7]

\begin{tabular}{|c|c|}
\hline Responsibility of the CKO & KMS Use Cases \\
\hline $\begin{array}{l}\text { Design, implement and supervise a } \\
\text { knowledge infrastructure for the company. }\end{array}$ & $\begin{array}{l}\text { Identify KM responsibilities based on some process breakdown } \\
\text { or segmentation. }\end{array}$ \\
\hline $\begin{array}{l}\text { Direct the development of a knowledge } \\
\text { strategy. }\end{array}$ & $\begin{array}{l}\text { Plan and schedule events. } \\
\text { Consult and report using data, information and knowledge on: }\end{array}$ \\
\hline $\begin{array}{l}\text { Provide critical input for the knowledge } \\
\text { and use creation process in the company }\end{array}$ & $\begin{array}{l}\text { plans, performance description, cause-effect analysis of } \\
\text { performance and forecasting and prediction of KM staff. }\end{array}$ \\
\hline $\begin{array}{l}\text { (new product development, market research } \\
\text { and strategic business development) and } \\
\text { efforts to improve those processes as }\end{array}$ & $\begin{array}{l}\text { Consult and report using data, information and knowledge on } \\
\text { evaluation of performance of KM staff in cost-benefit terms. }\end{array}$ \\
\hline required. & \\
\hline $\begin{array}{l}\text { Design and implement approaches in terms } \\
\text { of generating, coding and transferring }\end{array}$ & $\begin{array}{l}\text { training, conference, compensation and budget options in terms } \\
\text { of the anticipated cost benefit. }\end{array}$ \\
\hline knowledge to the company. & Specify and compare alternative options regarding levels of \\
\hline $\begin{array}{l}\text { Make all necessary economic } \\
\text { arrangements. }\end{array}$ & $\begin{array}{l}\text { effort, scope and content, Return On Inversion (ROI) objectives, } \\
\text { support for KM alternatives, support for KM staff, in terms of } \\
\text { the anticipated cost benefit. }\end{array}$ \\
\hline Provide the processes that & \\
\hline
\end{tabular}




\begin{tabular}{l|l}
\hline learning and sharing new knowledge. & Ease of communication, coordination and collaboration. \\
\hline $\begin{array}{l}\text { Manage relations with outside suppliers of } \\
\text { information and knowledge (academic } \\
\text { partners or data base companies) and } \\
\text { negotiate contracts with them }\end{array}$ & $\begin{array}{l}\text { Ease of communication, coordination and collaboration. } \\
\text { Specify and compare scope and content alternatives, ROI } \\
\text { objectives, support for KM alternatives, in terms of the } \\
\text { anticipated cost benefit. }\end{array}$ \\
\hline $\begin{array}{l}\text { Provide rewards and incentives to } \\
\text { contribute to the organizational knowledge } \\
\text { base. } \\
\begin{array}{l}\text { Set professional standards and manage the } \\
\text { careers of the knowledge managers. }\end{array}\end{array}$ & $\begin{array}{l}\text { Retrieve qualification information available on candidates. } \\
\text { Evaluate available candidates according to roles, relating } \\
\text { qualifications to predict performance. } \\
\text { Plan or select training plans. } \\
\text { Buy or create training methods and materials (seminars, } \\
\text { computer-based training, product, manuals, etc.). }\end{array}$ \\
\hline $\begin{array}{l}\text { Measure and manage the value of } \\
\text { knowledge. }\end{array}$ & $\begin{array}{l}\text { Measure knowledge use and re-use statistics } \\
\text { Analyze the direct commercial value of knowledge-intensive } \\
\text { business processes }\end{array}$ \\
\hline
\end{tabular}

Table 5. KMS use cases for supporting the CKO

Source: Adapted from [7]

\section{APPLICATION OF THE METHODOLOGICAL PROPOSAL TO THE CASE STUDY}

The Information Systems Research Laboratory (LISI) is a research group of Universidad Simón Bolívar (Venezuela), whose main interest is the study of technologies that support the development process of IS.

LISI currently has a working group made up of researchers and research assistants at different universities in the country, as well as support staff. LISI has relationship with the productive sector and has the technological platform necessary to carry out its research and development activities and attain its goals.

These R\&D activities involve the constant creation of knowledge that has to be available for transfer and use both by researchers and the productive sector related to LISI, and also to meet the growing need for fundamental collaboration, cooperation and communication in the interaction of its members.

In keeping with the development process put forward by the methodological proposal [10] the iteration plan for the Inception phase consisted of the activities listed in Table 6.

\section{INCEPTION PHASE}

1. Modeling the business.

a. Identifying the business use cases.

b. Identifying the actors.

c. Describing the use cases.

d. Preparing the use case model. 


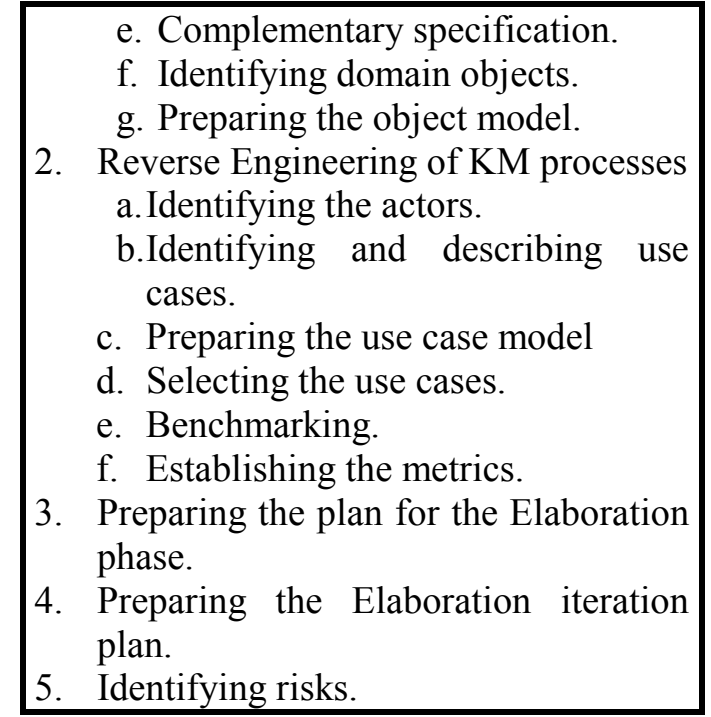

Table 6. Iteration plan for the Inception phase

These activities seek to achieve a common understanding of the business and establish the critical KM use cases with which the process will be started.

Five critical use cases were chosen from the results of this phase and an operational goal was set for the development process, as well as the metrics for ensuring its success.

After the Inception phase the use cases selected were redesigned in order to obtain a prototype of a beta version of the KMS and then propose architecture for the prototype developed. This was done during the Elaboration phase in which the activities listed in Table 7 were involved.

\section{ELABORATION PHASE}

1. Forward Engineering of the new KM processes

a. Identifying actors

b. Identifying use cases
c. Preparing the new use case model
d. Preparing the ideal object model
e. Preparing the real object model
f. Development of the project portfolio
g. Model of KMS use cases
h. Prototype of the KMS
i. Development of the Architecture
j. Selection of tools

2. Preparing the Construction phase plan.

3. Preparing the Construction iteration plan.

4. Reconsideration of risks.

Table 7. Iteration plan for the Elaboration phase

Note that the main activity in this phase consists of generating new processes from the existing ones, with the support provided by IT. This phase could therefore lead to new use cases or change the former ones.

A series of twelve use cases was one of the results of this phase, they were: evaluation and selection of technology, project management, selection and planning of training, virtual meetings, search and retrieval of data, information and knowledge, exchange with internal and external sources, support for users, updating of the knowledge base, discussion, updating of data and information, interaction with suppliers and, evaluation and selection of candidates.

These twelve use cases represent the redesign of the use cases selected in the previous phase (Figure 2). These use cases fall within the following areas: technological acquisition, project management, organizational memory, internal learning and external learning. 
Mainly they support the generation, coding and transfer of knowledge, though the updating of data, information and knowledge by the different users on the repositories and knowledge and discussion bases.

The role of the CKO must be determined so that relevant knowledge for the organization can be constantly evaluated so it is properly structured.

Likewise, a real object model was produced where the different control, entity and interface objects derived from the use case model mentioned earlier were represented (Figure 3). Those objects interact to comply with the objective of each use case (i.e. each KM process).

In the real object model (see Figure 3), objects related to the deployment environment are added to the ideal object model (created previously). The latter does not take these restrictions into account, whereas for the real one it was assumed that the use cases could not be implemented on any platform, except under in very specific circumstances [15].

The development of the portfolio of projects derived from the application of the second methodological phase is obtained by combining the objects of the previous model. This generates a series of system use cases, which are represented in the model shown in Figure 4. Those use cases represent a prescriptive model; they represent functional types of systems or tools that have to be included in the organization's KMS. Each of them, or the combination of some or all of them, will require a complete development process to reach a system that is custom built for the organization. So far one can see how to get to those systems from the knowledge processes that have become explicit during the modeling; since it guarantees effectiveness [11].

Next, the use cases that will be considered within the KMS were selected, as was their architecture. In the Construction phase, an initial version of them was obtained and the activities listed in Table 8 undertaken:

\section{CONSTRUCTION PHASE}

1. First version of the KMS

a. Development of Internet searches.

b. Development of User support.

c. Development of Messaging.

d. Development of a Project Management System.

e. Developing a Knowledge Base.

f. Developing Virtual Meetings.

2. Preparing the Transition phase plan.

3. Preparing the Transition iteration plan.

4. Reconsideration of risks.

Table 8. Iteration plan for the Construction phase

Lastly, during the Transition phase a final version of the KMS Use Cases selected was obtained and the implementation and training needed was undertaken. Table 9 shows the activities involved.

\section{TRANSITION PHASE}

1. Final version of KMS

a. Developing Internet searches

b. Developing user support

c. Developing messaging 

d. Developing a Project Management System.
e. Developing a Knowledge Base.
f. Developing Virtual Meetings.
g. Implementation of self-guided groups.
h. Nominal Group Techniques.
i. Face-to-face meetings.
j. Reward plan.
2. Reconsideration of Risks.

Table 9. Iteration plan for the Transition phase

The implementation of KMS included the following main options: Project Management, a Knowledge Base, User support, a Discussion base and a Decision Support System (DSS), as shown in the main interface (Figure 5).

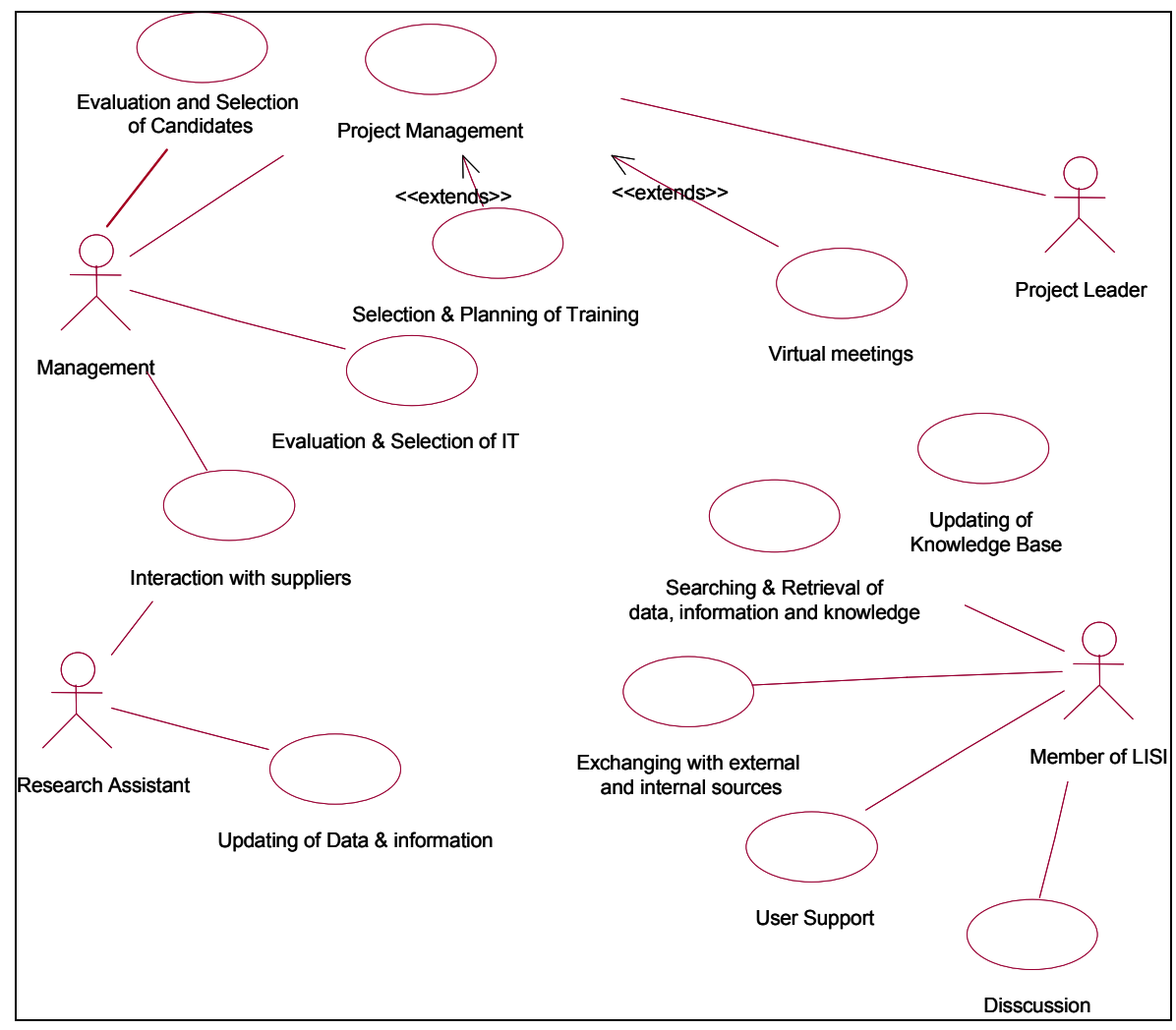

Figure 2. Model of Use Cases for LISI's new KM processes 


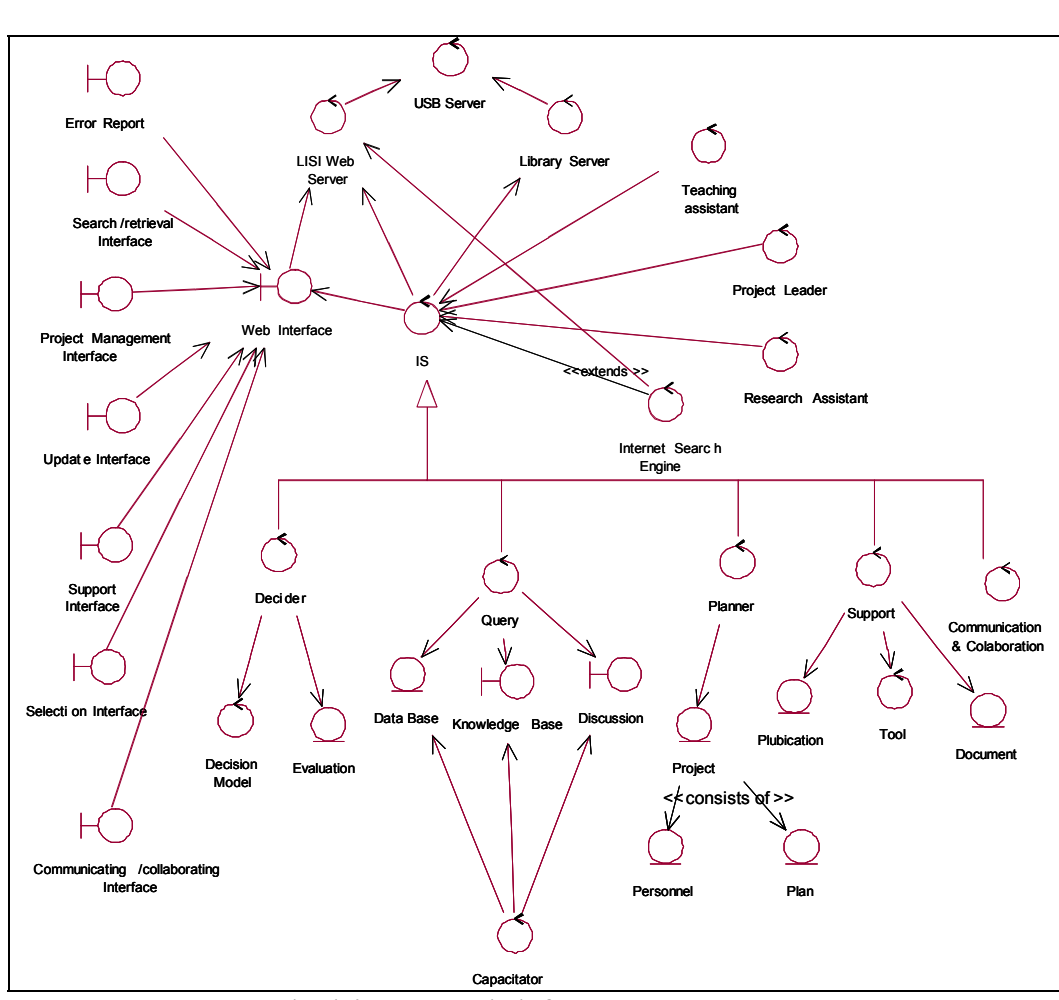

Figure 3. Real object model for LISI's new KM processes

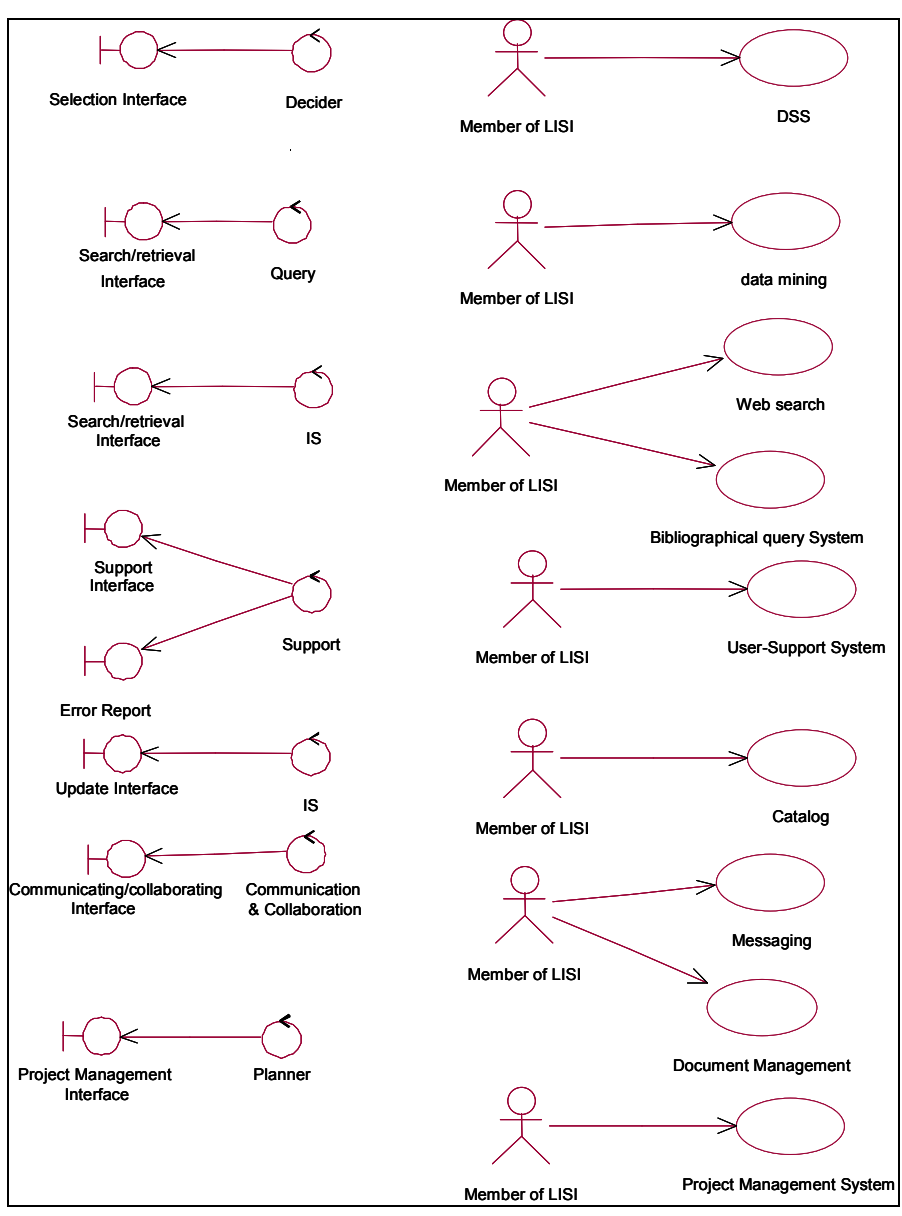

Figure 4. Use cases of systems 

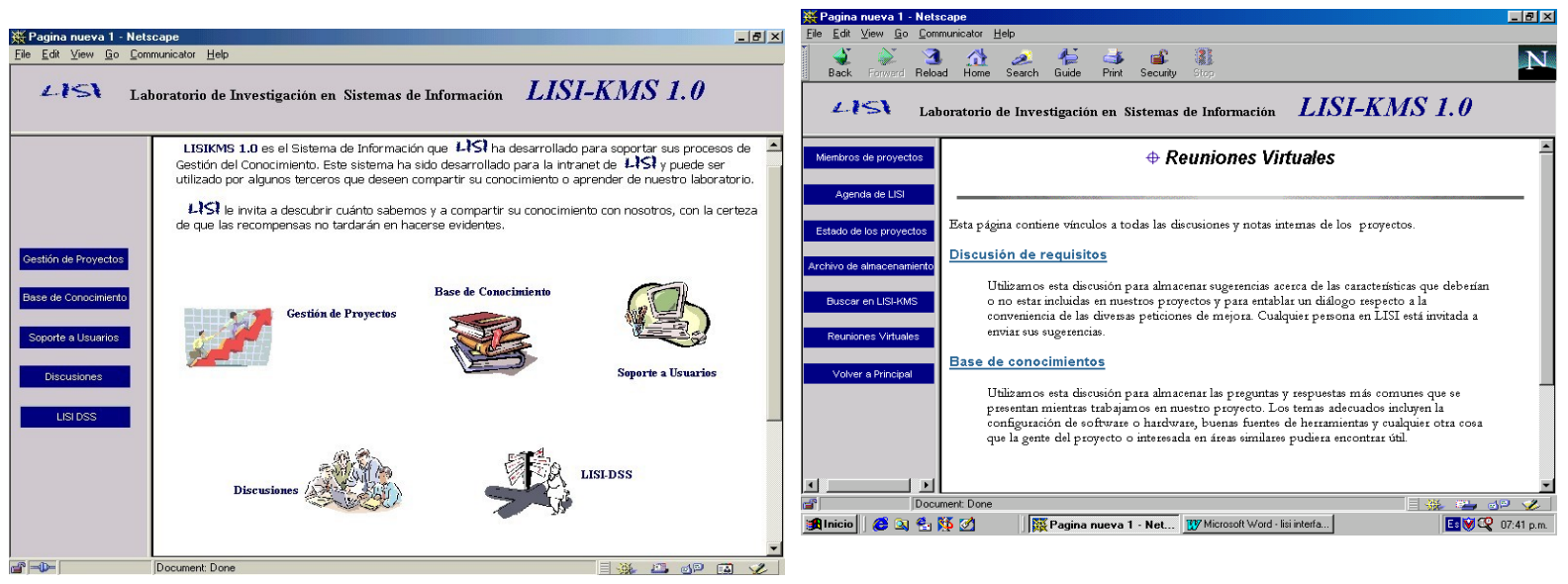

Figure 5(a). Interfaces of LISI-KMS
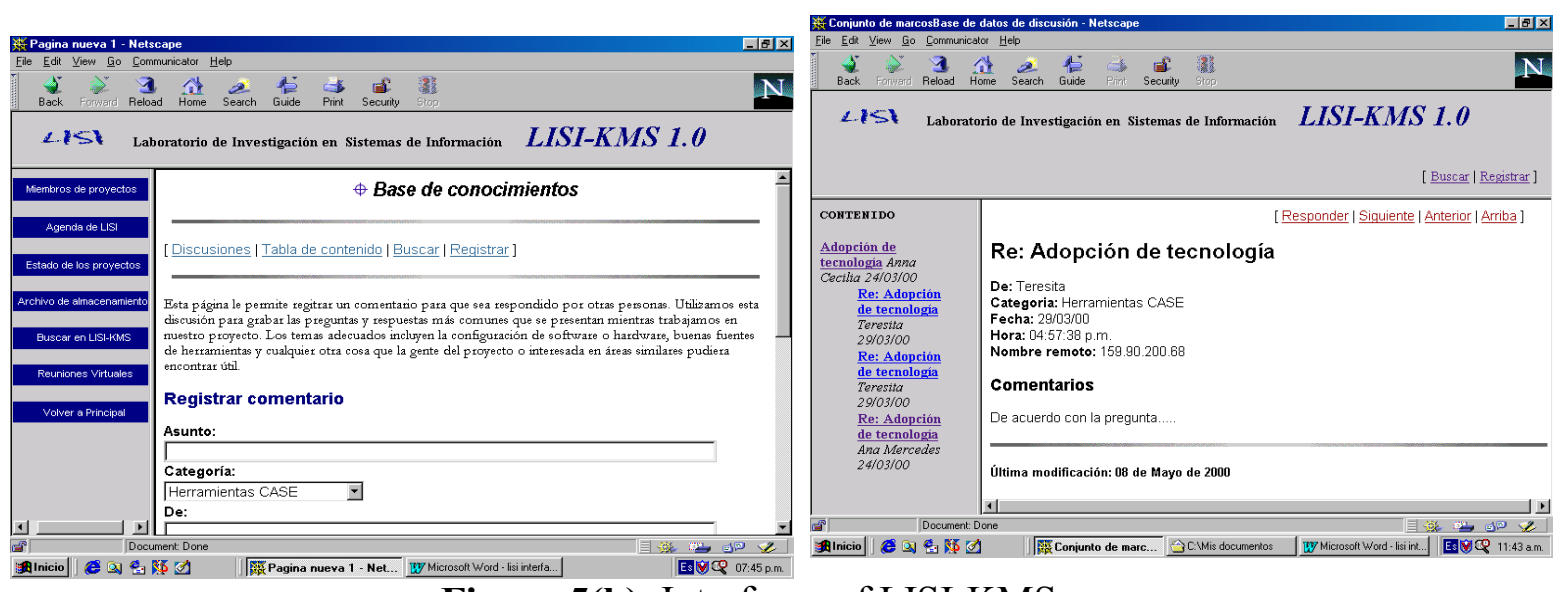

Figure 5(b). Interfaces of LISI-KMS

\section{ANALYSIS OF RESULTS}

The following analysis shows the main deliveries obtained from applying the methodological approach. To keep this document brief, only some of them were selected, including some deliveries not shown in this article [9].

- The business model: LISI's model of use cases of business processes was a useful tool for determining the dimensions of the complexity of the knowledge managed.

The technique was useful from the point of view of business knowledge, because of its acceptance and use, which gave LISI's staff a share vision of its business model.

- The Vision: application of the methodological proposal showed that it is not easy to establish a vision that expresses quantitatively what is expected of the KM project. The reason is simple: since much of the knowledge 
is hard to find and capture, it is difficult to measure the percentage of total knowledge coded and available. The metrics established for evaluating the vision must be considered and revised in the medium and long term (in a 3 to 5 year horizon) since prior to that, how to establish the degree of effectiveness is not obvious. This coincides with what Hartz et al. [13] have said. However, a good way of guaranteeing this effectiveness is through constant monitoring of the critical success factors proposed.

- The model of existing KM processes: this model shows a set of nine use cases that can be interpreted as the ways in which an organization uses Knowledge Management processes. In the case of LISI it was hard to pinpoint these use cases, due mainly to the fact that some of them have very similar functions to one another. Each use case can be associated with one or more organizational areas. Graphic representation facilitates visualization of the interactions between areas that the KMS must solve and support.

- The model of new KM processes: this model covers several of the existing use cases and retains others that have undergone changes in the course of events. It also adds new use cases that represent new ways of using the KM processes.

- The real object model of the new KM processes: enables control of the solution for KM processes to be established. By including the implementation aspects, the organization's restrictions in handling such processes are clearly identified.

- The IS use case model: this model was extremely useful as it used active objects to show the different actors' views and the functionalities that respond to them. This model was essential in defining the project portfolio subsequently prepared through LISI-KMS.

- List of risks: A review of the risks in each phase and their mitigation was an important factor as there are many human, technological, financial, management or organizational risks, all capable of leading to failure faster than they would in projects of a different kind. This is very significant in this type of project. In this experience in particular, in addition to the traditional risks inherent in planning, analysis, design, execution and implementation activities, people's resistance to technology, collaboration, cooperation and willingness to share knowledge are considered risks of human and social nature, related to a reluctance to change. These risks have a high value within the scale of impact. It was therefore necessary to prepare a mitigation plan, based on motivation, promotion and reward for sharing knowledge. The group's techniques and dynamics were included in this plan with a view to narrowing friction and fostering the right climate and culture.

- The project plan: this product is extremely useful to determine 
Management's commitment to the project. Particularly in the case of LISI, the result of planning the project is merely a formality, given time and resource constraints. Hence it does not provide much information on estimates, which in any other case would be decisive in determining whether to continue or cancel the project.

Figure 6 shows the relationship between these results and the methodological proposal applied.

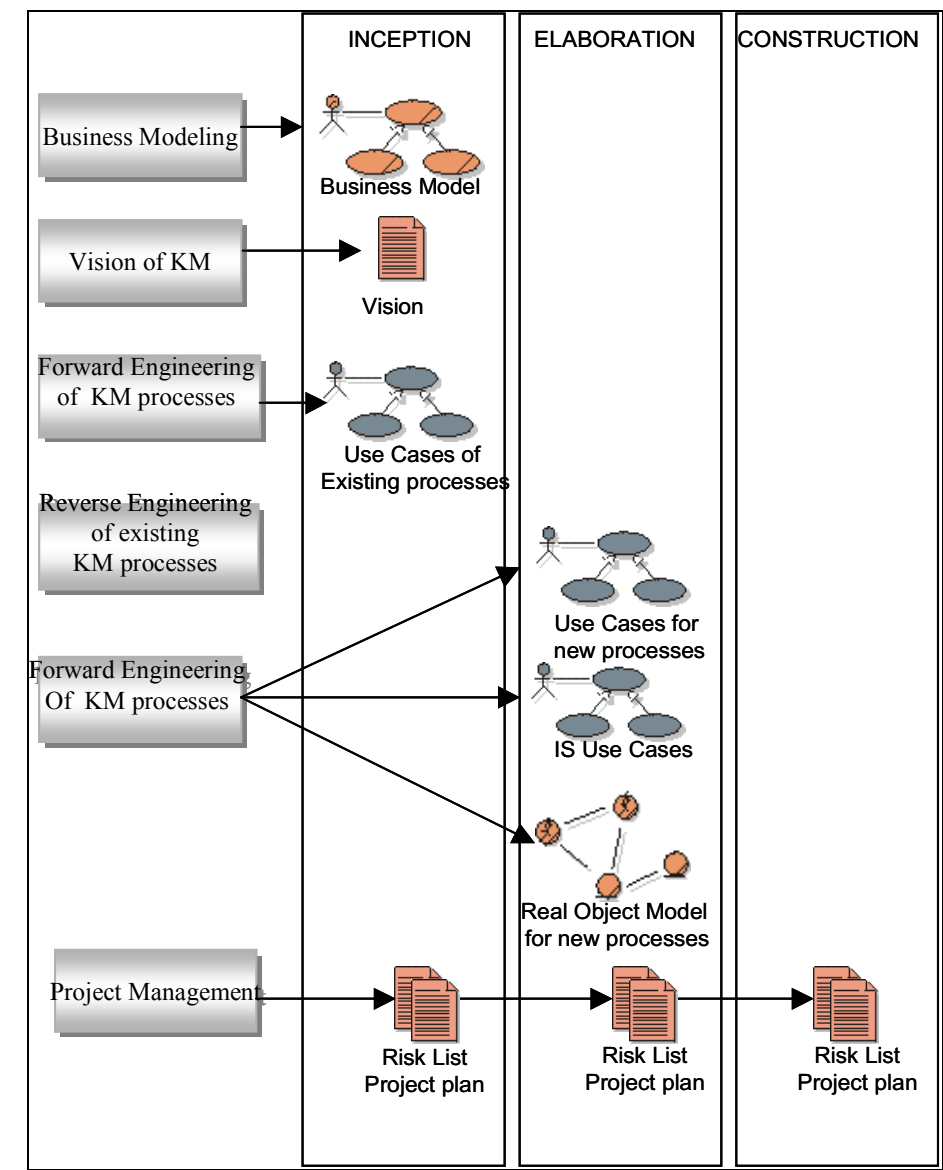

Figure. 6. Relationship between the results analyzed and the methodology applied

\section{LESSONS LEARNED}

Once the results of applying the methodological approach to the case study were analyzed, the following lessons on the process itself were obtained:
As a central aspect, the KMS development process must take into account the existing $\mathrm{KM}$ processes in order for them to be consistent with the idea that there is a natural $\mathrm{KM}$ in the organization, while the support of IT is fostered through the development of a 
KMS and a portfolio of related projects that can be integrated.

In applying a systemic approach, the organization was seen as a whole, made up of different subsystems, including the social and technical one, which guarantees the investment in terms of effort and resources in those critical success factors linked to each of these aspects. Likewise, applying an iterative process enables the objective to be approached gradually and enhances the quality of the results.

The proposal for using objectorientation and the use case technique makes it possible to have an intuitive annotation with a semantic richness that makes it easier to reach a common understanding.

Lastly, in the domain of $\mathrm{KM}$, it is essential that the development process emphasis risk and change management..

A series of topics also arose from learning this application:

- In the Business Model, preparation and analysis of business objects was included. It was decided to use: the Business Object Model and a Glossary of them.

- In the Reverse Engineering of the existing KM processes, as well as in the Forward Engineering of new processes, each one's relationship with the business units (subsystems) was included.

- A level of complexity of no more than 10 use cases per model was established, where each model must be balanced against the corresponding Object Model or user interface prototype.

- The document Supplementary KM specifications was included. It can be used to specify important information that could not be modeled due to annotation constraints.

- In the Forward Engineering of new KM processes, Interaction Diagrams for each use case were included. Thus it was possible to show how objects within each of them communicate and collaborate with one another.

\section{CONCLUSIONS AND RECOMMENDATIONS}

The methodological aspects proposed show that it is feasible to consider developing a KMS based on existing $\mathrm{KM}$ processes, since its effectiveness depends on the characteristics shown by the context, as far as generating, assigning and applying knowledge are concerned.

Application of this methodological proposal revealed the combination of the three fundamental aspects on which the success of a KM initiative depends, through such factors as: management's commitment to knowledge management, the organization's openness, inter-connected structure, availability of resources, size and nature of the business, full interaction with the people who have the knowledge to be coded, organizational culture reflected by the willingness of its professionals to generate and share knowledge. 
The methodological approach used proposes: (1) a set of UML models for specifying knowledge processes and the KM architecture to be implemented; (2) a semantic way of arriving at the systems portfolio necessary to support the existing knowledge processes; (3) an iterative development process that combines 7 workflows and 4 phases to go from the design stage of the business and the vision of KM up to specific applications of KMS.

Application of this proposal to the case study enabled it to be refined. Repeating this experience in other organizations, in different contexts, involving other existing methodologies, will help to achieve better levels of learning. It is also necessary to explore other integration technologies that enable KMS to be expanded.

Lastly, the results obtained and validated here can become part of the experience and practice base of any Learning Software Organization (LSO) interested in participating in and developing IT intensive KM initiatives. According to Ruhe [24] a LSO is an organization that learns within the domain of software development, evolution and application. In the context of LSO, KM and learning approaches are complementary views on knowledge handling processes.

\section{BIBLIOGRAPHICAL REFERENCES}

[1] R. Alterman, Panel discussion on Case Representation, in: Second
Workshop on Case-Based Reasoning, Pensacola Beach, USA, 1989, Web publication available via: www.boffin.ucd.ucd.ie/ulysses/ CBR/Watson/CBR-biblWatson.htm.

[2] I. Becerra-Fernandez and R. Sahherwall, Organizational Knowledge Management: A Contingency Perspective, Journal of Information Management. Systems (18:1), 2001 pp. 23--55.

[3] E. Conklin, Designing Organizational Memory: preserving intellectual assets in a knowledge economy, Web publication available via: www.zilker.net/business/info/pubs/d esom/body.htm, 1996.

[4] M. Creemers, F. Derksen and M. Huysman, Learning from the Environment: Exploring the Relation Between Organizational Learning, Knowledge Management and Information /Communication Technology, in: Fourth Americas Conference on Information Systems, Association on Information Systems ed., USA, 1998, pp. 598--600.

[5] T. Davenport and L. Prusak, Working Knowledge, Harvard Business Scholl Press, USA, 1998, pp. 19-106.

[6] B. David, Principles for Case Representation in a case-based aiding system for lesson planning, in: Workshop on Case-based Reasoning, Washington, USA, 1991, pp. 351--362.

[7] J. Firestone, Enterprise Knowledge 
Management Modeling and Distributed Knowledge Management Systems. Executive Information Systems, Inc. White paper No. 12, 1999. Available on: http://www.dkms.com/EKMDKMS. html

[8] A. Gold, A. Malhotra and A. Segars, Knowledge Management: An Organizational Capabilities Perspective, Journal of Information Management. Systems, (18:1), 2001, pp. 185--213.

[9] A. Grimán, Propuesta Metodológica Sistémica para la Gestión del conocimiento dentro de las organizaciones. Msc. Thesis, Universidad Simón Bolívar, 2000.

[10] A. Grimán, M. Pérez and T. Rojas, A Systemic Methodological Proposal for Knowledge Management in organizations, in: World Multiconference on Systemics, Cybernetics and Infor-matics, Vol. 10, Callaos y Callaos eds. Orlando Florida, 2000, pp. 32--37.

[11] V. Grover and T. Davenport, General Perspective on Knowledge Management: Fostering a research Agenda, Journal of Information Management Systems, (18:1), 2001, pp. 5--21.

[12] V. Gupta and I. Yermish, An Aproach to Knowledge Management: The Contribution of Technical and Social Concepts, in: Fourth Americas Conference on Information Systems, Association on Information Systems ed., USA, 1998, pp. 585--587.

[13] C. Hartz, S. Sammis, J. HoferAlfeis, K. Lopez, C. Raybourn and J. Neumann, American Productivity \& Quality Center, Web Publication available via: http://www.apqc.org/free/articles/ dispArticle.cfm?ProductID $=1307$, 2001.

[14] T. Housell and A. Bell, Measuring and managing knowledge, $\mathrm{Mc}$ Graw-Hill, NY, USA, 2001.

[15] I. Jacobson, M. Ericsson and A. Jacobson, The object advantage. Business Process Reengineering with object technology, ACM Press, USA, 1995.

[16] J. Kolodner, Case-based Reasoning, Moegan Kaufmann, USA, 1993.

[17] P. Kruchten, The Rational Unified Process, Addison Wesley Longman, Inc., USA, 1999, pp. 41--60.

[18] J. Laudon and K. Laudon, Management Information Systems, Prentice Hall, USA, 2000, pp. 33--34.

[19] Y. Malhotra, Knowledge Management for the New World of Business, Web publication available via www.brint.com $/ \mathrm{km} /$ whatis.htm, @Brint.com BizTech Network.

[20] M. Mc Lure, A framework for successful Knowledge Mana- 
gement Implementation, in: Fourth Americas Conference onInformation Systems, Association on Information Systems ed., USA, 1998, pp. 635-637.

[21] Microsoft Solution Framework, Web publication available via: www.microsoft.com $/ \mathrm{msf} /$ models.h tml, Microsoft Corporation.

[22] J. O'Brien, Introduction to Information Systems, Irwin Book Team, USA, 1999, pp. 538--539.

[23] C. Riesbeck, Inside Case-based Reasoning, Lawrence Erlbaum Associates, Inc., USA., 1989.

[24] G. Ruhe, Learning Software Organization, in: Handbook of Software Engineering and Knowledge Engineering (SK. Chang, ed.). World Scientific Publishing Co., Vol. 1, 2001, pp. 399--419.
[25] E. Stein, Organizational memory: Review of concepts and recommendations for management, International Journal of Information Management, (15:1), 1995, pp. 17--32.

[26] T. Stewart, Intellectual Capital, Doubleday/Currency, NY, USA, 1997, pp. 167--198.

[27] K. Sveiby, What is Knowledge Management?, Web publication available: www.sveiby.com.au.

[28] E. Turban et al., Information Technology for Management. Making connections for Strategic Advantage. 2nd. Ed. John Wiley \& sons inc., NY, 1999, pp. 338-340 .

[29] J. Walsh and G. Ungson, Organizational Memory, Academy of Management Review, (16:1), 1991, pp. 57--91. 